\title{
Function Spaces as Dirichlet Spaces (About a Paper by Maz'ya and Nagel)
}

\author{
Niels Jacob and René L. Schilling
}

\begin{abstract}
V. G. Maz'ya and J. Nagel found for certain classes of weighted Sobolev norms (defined using the Fourier transform) equivalent Slobodeckij-type difference representations. We extend these considerations to a wider class of anisotropic norms which arise in the theory of Markov processes. In particular we show that these Sobolev norms are equivalent to Dirichlet norms.

Keywords: Dirichlet space, anisotropic Sobolev space, Slobodeckij norm, negative definite function, Bernstein function
\end{abstract}

MSC 2000: 46E35, 31C25, 26A99, 42B35

\section{Introduction}

In [18] V. G. Maz'ya and J. Nagel considered anisotropic function spaces $H^{\mu}\left(\mathbb{R}^{n}\right)$ which are defined as completion of the test functions $C_{c}^{\infty}\left(\mathbb{R}^{n}\right)$ with respect to the norm

$$
\|u\|_{H^{\mu}}^{2}:=\int_{\mathbb{R}^{n}}|\widehat{u}(\xi)|^{2} \mu(\xi) d \xi+\int_{\mathbb{R}^{n}}|\widehat{u}(\xi)|^{2} d \xi,
$$

where $\mu(\xi)=\sum_{j=1}^{n} \mu_{j}\left(\left|\xi_{j}\right|\right)$ and $\mu_{j}: \mathbb{R} \rightarrow \mathbb{R}$ are temperate weight functions in the sense of Hörmander.* The aim of Maz'ya and Nagel was to find a norm equivalent to (1) but one which involves integrals of differences of functions and avoids the Fourier transform, i.e.,

$$
\mathbf{|} u \mathbf{|}_{H^{\mu}}^{2}=\int_{\mathbb{R}^{n}}\left(\int_{\mathbb{R}^{n}}|u(x+y)-u(x)|^{2} N(y) d y\right) d x+\int_{\mathbb{R}^{n}}|u(x)|^{2} d x
$$

N. Jacob: Department of Mathematics, University of Wales Swansea, Singleton Park, Swansea SA2 8PP, U.K.; n.jacob@swansea.ac.uk

R. L. Schilling: FB 12 Mathematik, Philipps-Universität Marburg, D-35032 Marburg, Germany; schilling@mathematik.uni-marburg.de

* For reasons that will become clear later, we use here and in the sequel $\mu, N$ and $g$ rather than $\mu^{2}, N^{2}$ and $g^{2}$ as in the original papers. This is no loss of generality since $\mu^{2}$ is a temperate weight function whenever $\mu$ is.

ISSN 0232-2064 / \$2.50 (c) Heldermann Verlag Berlin 
where $N(y)$ is a weight function* depending on the $\mu_{j}\left(\left|\xi_{j}\right|\right), 1 \leqslant j \leqslant n$. In [19] Nagel considered rotationally invariant temperate weights $\mu=\mu\left(|\xi|^{2}\right)$ which under some additional conditions - lead to equivalent norms of the form

$$
\mathbf{|} u \mathbf{|}_{H^{\mu}}^{2}=\int_{\mathbb{R}^{n}}\left(\int_{\mathbb{R}^{n}}|u(x+y)-u(x)|^{2} g\left(\frac{1}{|y|^{2}}\right) \frac{d y}{|y|^{n}}\right) d x+\int_{\mathbb{R}^{n}}|u(x)|^{2} d x,
$$

where $g$ and $\mu$ are related through

$$
\mu(t)=\int_{0}^{t}\left(\int_{r}^{\infty} g(s) \frac{d s}{s^{2}}\right) d r
$$

The interesting observation is that (2) and (3) are norms in Hilbert spaces which are invariant under contractions.

Definition 1.1. A Hilbert space $(\mathfrak{H},\langle\bullet, \bullet\rangle)$ of real functions satisfies the contraction property if for every $u \in \mathfrak{H}$ the function $w:=0 \vee u \wedge 1 \in \mathfrak{H}$ and $\langle w, w\rangle \leqslant\langle u, u\rangle$.

Since the norms (2) or (3) are invariant under translations, a result of A. Beurling and J. Deny [6], see also [16], shows that the associated function spaces are Dirichlet spaces and that the weight function $\mu(\xi)$ for the norm (1) can be chosen to be a continuous negative definite function $\psi(\xi)=\mu(\xi)$; this choice achieves not only equivalence but equality between (1) and (3). Incidentally, the spaces $\left(H^{\psi},\|\cdot\|_{H^{\psi}}\right)$ were considered by Jacob [16] within a larger scale of anisotropic Sobolev spaces $H^{\psi, s}, s \in \mathbb{R}$, where $H^{\psi, s}=H^{(1+\psi)^{s}}$ if $s>0$. This scale plays a major role in the construction of Markov processes which are generated by pseudo differential operators.

The aim of our paper is to understand the connection between $\mu$ resp. $\psi$ and $g$ in the rotationally invariant situation. It turns out that subordination in the sense of Bochner is the key to understanding this relation. Our main result Theorem 4.5 states that under some conditions on $g$ (or $\mu$ ) it is possible to determine a Bernstein function $f$ such that (3) is equivalent to

$$
\int_{\mathbb{R}^{n}}|\widehat{u}(\xi)|^{2} f\left(|\xi|^{2}\right) d \xi+\int_{\mathbb{R}^{n}}|\widehat{u}(\xi)|^{2} d \xi
$$

(Note that $\xi \mapsto f\left(|\xi|^{2}\right)$ is a continuous negative definite function.)

In Section 2 we collect some definitions and results on negative definite functions, Bernstein functions and subordination. Section 3 contains some auxiliary results and mainly technical calculations which we will need for the main result Theorem 4.5 in Section 4. For our considerations we need to know the structure of continuous negative functions which are invariant under rotations. Such characterizations are (partly) known, but they are somewhat hidden in the papers by Schoenberg [23] and Kahane [17]. In order to be self-contained we give a new proof for this in the appendix where we also tabulate examples of (complete) Bernstein functions and their representation measures. 


\section{Preliminaries}

A temperate weight function is a positive function $\mu: \mathbb{R}^{n} \rightarrow \mathbb{R}^{+}$such that for positive constants $C$ and $N$

$$
\mu(\xi+\eta) \leqslant(1+C|\xi|)^{N} \mu(\eta), \quad \xi, \eta \in \mathbb{R}^{n} .
$$

It is easy to see that sum or product of two temperate weight functions are again temperate weight functions, cf. Hörmander $[9, \S 2]$ or $[10, \S 10]$ for this and further results on weight functions.

Real-valued negative definite functions $\psi: \mathbb{R}^{n} \rightarrow \mathbb{R}$ will be central to our considerations. These are functions such that the matrix

$$
\left(\psi\left(\xi^{j}\right)+\psi\left(\xi^{k}\right)-\psi\left(\xi^{j}-\xi^{k}\right)\right)_{j, k=1}^{N}
$$

is positive semidefinite for any choice of $N \in \mathbb{N}$ and $\xi^{1}, \xi^{2}, \ldots, \xi^{N} \in \mathbb{R}^{n}$. It is important to note that a negative definite function is not "minus" some positive definite function (in the sense of Bochner), but that $\psi: \mathbb{R}^{n} \rightarrow \mathbb{R}$ is negative definite if, and only if, for all $N \in \mathbb{N}$ and $\xi_{1}, \xi_{2}, \ldots, \xi_{N} \in \mathbb{R}^{n}$

$$
\psi(0) \geqslant 0 \quad \text { and } \quad \sum_{j, k=1}^{N} \psi\left(\xi_{j}-\xi_{k}\right) \lambda_{j} \bar{\lambda}_{k} \leqslant 0 \quad \text { for all } \quad \lambda_{j} \in \mathbb{C}, \quad \sum_{j=1}^{N} \lambda_{j}=0 .
$$

Equivalently, $\psi$ is negative definite if, and only if,

$$
\psi(0) \geqslant 0 \quad \text { and } \quad \xi \mapsto e^{t \psi(\xi)} \quad \text { is positive definite for all } t>0 \text {. }
$$

Moreover, $\psi: \mathbb{R}^{n} \rightarrow \mathbb{R}$ is continuous and negative definite (we use c.n.d.f. as a shorthand) if, and only if, the following Lévy-Khinchine representation holds

$$
\psi(\xi)=c_{0}+\sum_{j, k=1}^{n} q_{j k} \xi_{j} \xi_{k}+\int_{y \neq 0}(1-\cos y \xi) \nu(d y)
$$

where $c_{0}=\psi(0) \geqslant 0,\left(q_{j k}\right)_{j, k} \in \mathbb{R}^{n \times n}$ is a symmetric positive semidefinite matrix and $\nu$ is a Radon measure on $\mathbb{R}^{n} \backslash\{0\}$ such that $\int_{y \neq 0}\left(|y|^{2} \wedge 1\right) \nu(d y)<\infty$. The measure $\nu$ is called Lévy measure. For proofs and a more detailed discussion we refer to the monograph by C. Berg and G. Forst [4, $\S \S 7-8]$ or [16].

Using the very definition of negative definite functions it is possible to show that $\xi \mapsto \sqrt{\psi(\xi)}$ is subadditive and, cf. [16, Lemma 3.6.25], that the following Peetre-type inequality holds

$$
1+\psi(\xi+\eta) \leqslant(1+\sqrt{\psi(\eta)})^{2}(1+\psi(\xi)), \quad \xi, \eta \in \mathbb{R}^{n} .
$$


This means, in particular, that every real-valued continuous negative definite function $\psi$ is a temperate weight function.

A $C^{\infty}$-function $f:(0, \infty) \rightarrow \mathbb{R}$ is said to be completely monotone resp. a Bernstein function, if

$$
(-1)^{k} \frac{d^{k} f(x)}{d x^{k}} \geqslant 0 \quad \text { resp. } \quad f \geqslant 0, \quad(-1)^{k+1} \frac{d^{k+1} f(x)}{d x^{k+1}} \leqslant 0
$$

holds for all $k \in \mathbb{N}_{0}$. A well-known theorem of $\mathrm{S}$. Bernstein states that all completely monotone functions are of the form

$$
f(x)=\int_{0}^{\infty} e^{-x s} \sigma(d s),{ }^{\dagger} \quad x>0,
$$

with a uniquely determined finite measure $\sigma$ on $[0, \infty)$. Completely monotone and Bernstein functions are in many points similar to positive and negative definite functions. In fact, completely monotone resp. Bernstein functions are the positive resp. negative definite functions in the additive semigroup $(0, \infty)$. In particular, $f:(0, \infty) \rightarrow \mathbb{R}$ is a Bernstein function if, and only if,

$$
f(0+) \geqslant 0 \quad \text { and } \quad x \mapsto e^{-t f(x)} \quad \text { is completely monotone for all } t>0 .
$$

Moreover, the Lévy-Khinchine representation

$$
f(x)=a+b x+\int_{0+}^{\infty}\left(1-e^{-x s}\right) \tau(d s)
$$

gives a one-to-one correspondence between the Bernstein functions $f$ and triplets $(a, b, \tau)$ where $a, b \geqslant 0$ and $\tau$ is a Radon measure on $(0, \infty)$ such that $\int_{0+}^{\infty}(s \wedge$ 1) $\tau(d s)<\infty$. We need to consider the class of complete Bernstein functions (also known as operator-monotone functions) which are Bernstein functions whose representing measure is of the form $\tau(d s)=m(s) d s$ with a completely monotone density $m(s)$. Equivalently, $f$ is a complete Bernstein function if, and only if,

$$
f(x)=a+b x+\int_{0+}^{\infty} \frac{x}{t+x} \rho(d t),
$$

where $\rho$ is a Radon measure on $(0, \infty)$ such that $\int_{0+}^{\infty}(1+t)^{-1} \rho(d t)<\infty$. Note that (13) means that the function $x \mapsto f(x) / x$ is a Stieltjes transform, cf. [4, $\S 17]$. Examples of (complete) Bernstein functions are listed in Appendix 1.

If $\psi$ is a c.n.d.f. and $f$ is a Bernstein function, then $f \circ \psi$ is again continuous and negative definite, cf. [16, Lemma 3.9.9]. We call $f \circ \psi$ the continuous negative definite function subordinate to $\psi$ with respect to $f$.

From (9) it is obvious that $\xi \mapsto|\xi|^{2}$ is a c.n.d.f. The following result characterizes all continuous negative functions which are subordinate to $|\xi|^{2}$.

\footnotetext{
†We use the convention that $\int_{a}^{b}=\int_{[a, b)}$ and $\int_{a+}^{b}=\int_{(a, b)}$
} 
Lemma 2.1. A continuous negative definite function $\psi: \mathbb{R}^{n} \rightarrow \mathbb{R}$ is subordinate to the function $\xi \mapsto|\xi|^{2}$ if, and only if,

$$
\psi(\xi)=\psi(0)+b|\xi|^{2}+\int_{y \neq 0}(1-\cos y \xi) m\left(|y|^{2}\right) d y,
$$

where $b \geqslant 0$ and $m$ is the Laplace transform

$$
m(r)=\int_{0}^{\infty} e^{-r s} \nu(d s), \quad r>0
$$

of a measure $\nu$ on $(0, \infty)$ such that $\int_{0+}^{1} s^{-n / 2} \nu(d s)+\int_{1}^{\infty} s^{-n / 2-1} \nu(d s)<\infty$. The Bernstein function $f$ such that $\psi(\xi)=f\left(|\xi|^{2}\right)$ holds is given by

$$
f(x)=\psi(0)+b x+\int_{0+}^{\infty}\left(1-e^{-x s}\right)(4 \pi s)^{n / 2} \Phi(\nu)(d s),
$$

where $\Phi(\nu)(d s)=\nu\left(\Phi^{-1}(d s)\right)$ is the image measure of $\nu$ under $\Phi(s)=(4 s)^{-1}$, $s>0$.

A proof of Lemma 2.1 can be found in Appendix 2.

Not every rotationally invariant negative definite function $\psi: \mathbb{R}^{n} \rightarrow \mathbb{R}$ is of the form $\psi(\xi)=f\left(|\xi|^{2}\right)$ where $f$ is a Bernstein function. However, if we can define $\psi$ on every space $\mathbb{R}^{m}, m \in \mathbb{N}$, and if it is rotationally invariant, then $\psi(\xi)$ is necessarily of the form $f\left(|\xi|^{2}\right)$, and $f$ must be a Bernstein function. This is the essence of the following theorem which is known, but appears in somewhat hidden form in the papers by Kahane [17] and Schoenberg [23]. For the readers' convenience we give a new proof in Appendix 2.

Theorem 2.2. Let $\psi: \mathbb{R}^{\mathbb{N}} \rightarrow \mathbb{R}$ be a function such that for every $n \in \mathbb{N}$ the function $\left(\xi_{1}, \ldots, \xi_{n}\right) \mapsto \psi\left(\xi_{1}, \ldots, \xi_{n}, 0, \ldots\right)$ is negative definite and invariant under rotations. Then there exists a unique Bernstein function $f$ such that $\psi(\xi)=f\left(|\xi|^{2}\right)$ for all $\xi \in \mathbb{R}^{\mathbb{N}}$ with finitely many non-zero entries.

\section{Auxiliary Results}

We will need the following auxiliary theorems and technical results for the proof of the norm-equivalences in Section 4.

Lemma 3.1. Let $\psi(\xi)=\int_{y \neq 0}(1-\cos y \xi) \nu(d \xi)$ be a continuous negative definite function. Then

$$
\int_{\mathbb{R}^{n}}|\widehat{u}(\xi)|^{2} \psi(\xi) d \xi=\frac{1}{2} \iint_{\mathbb{R}^{n} \times\left(\mathbb{R}^{n} \backslash\{0\}\right)}|u(x+y)-u(x)|^{2} d x \nu(d y)
$$

holds for all $u \in \mathcal{S}\left(\mathbb{R}^{n}\right)$. 
Proof. Denote by $T_{-y}$ the translation operator $T_{-y} u(x):=u(x+y)$. Since $\left|e^{i t}-1\right|^{2}=2(1-\cos t), t \in \mathbb{R}$, we find using Plancherel's Theorem

$$
\begin{aligned}
\frac{1}{2} \iint_{\mathbb{R}^{n} \times\left(\mathbb{R}^{n} \backslash\{0\}\right)}|u(x+y)-u(x)|^{2} d x \nu(d y) \\
=\frac{1}{2} \int_{\mathbb{R}^{n} \backslash\{0\}}\left\|T_{-y} u-u\right\|_{L^{2}}^{2} \nu(d y) \\
=\frac{1}{2} \int_{\mathbb{R}^{n} \backslash\{0\}}\left\|\left(T_{-y} u\right)^{\widehat{u}}-\widehat{u}\right\|_{L^{2}}^{2} \nu(d y) \\
=\frac{1}{2} \int_{\mathbb{R}^{n} \backslash\{0\}}\left(\int_{\mathbb{R}^{n}}\left|\widehat{u}(\xi) e^{-i y \xi}-\widehat{u}(\xi)\right|^{2} d \xi\right) \nu(d y) \\
=\frac{1}{2} \int_{\mathbb{R}^{n}}\left(|\widehat{u}(\xi)|^{2} \int_{\mathbb{R}^{n} \backslash\{0\}}\left|e^{-i y \xi}-1\right|^{2} \nu(d y)\right) d \xi \\
=\int_{\mathbb{R}^{n}}\left(|\widehat{u}(\xi)|^{2} \int_{\mathbb{R}^{n} \backslash\{0\}}(1-\cos y \xi) \nu(d y)\right) d \xi \\
=\int_{\mathbb{R}^{n}}|\widehat{u}(\xi)|^{2} \psi(\xi) d \xi .
\end{aligned}
$$

If $\psi(\xi)=f\left(|\xi|^{2}\right)$, we see from Lemma 2.1 that $\nu(d y)=m\left(|y|^{2}\right) d y$ with a completely monotone density $m(r)$ and

$$
\int_{\mathbb{R}^{n}}|\widehat{u}(\xi)|^{2} f\left(|\xi|^{2}\right) d \xi=\frac{1}{2} \iint_{\mathbb{R}^{n} \times \mathbb{R}^{n}}|u(x+y)-u(x)|^{2} m\left(|y|^{2}\right) d y d x .
$$

Up to notational changes, the following result is proved in Maz'ya and Nagel [18].

Lemma 3.2. Let $g_{j}:[0, \infty] \rightarrow[0, \infty], 1 \leqslant j \leqslant n$, be monotone increasing functions such that

$$
\int_{0}^{1} \frac{g_{j}(s)}{s} d s+\int_{1}^{\infty} \frac{g_{j}(s)}{s^{2}} d s<\infty .
$$

Then $\mu_{j}(|t|):=\int_{0}^{|t|} \int_{r}^{\infty} g_{j}(s) / s d s d r$ is well-defined and

$$
\begin{gathered}
\sum_{j=1}^{n}\left(\iint_{\mathbb{R}^{1} \times \mathbb{R}^{n}}\left|u\left(x+t_{j} e_{j}\right)-u(x)\right|^{2} g_{j}\left(\frac{1}{t_{j}^{2}}\right) \frac{d x d t_{j}}{\left|t_{j}\right|}\right)^{\frac{1}{2}} \\
\sim\left(\int_{\mathbb{R}^{n}}|\widehat{u}(\xi)|^{2} \sum_{j=1}^{n} \mu_{j}\left(\left|\xi_{j}\right|^{2}\right) d \xi\right)^{\frac{1}{2}}
\end{gathered}
$$

are equivalent seminorms on $\mathcal{S}\left(\mathbb{R}^{n}\right)$. 
Nagel discussed in $[19,20]$ the rotationally invariant analogue of Lemma 3.2.

Lemma 3.3. Let $g:[0, \infty] \rightarrow[0, \infty]$ be a monotone increasing concave function and define $\mu(t):=\int_{0}^{t} \int_{r}^{\infty} g(s) / s^{2} d s d r \in[0, \infty]$. Then

$$
\begin{gathered}
\left(\iint_{\mathbb{R}^{n} \times \mathbb{R}^{n}}|u(x+y)-u(x)|^{2} g\left(\frac{1}{|y|^{2}}\right) \frac{d x d y}{|y|^{n}}\right)^{\frac{1}{2}} \\
\sim\left(\int_{\mathbb{R}^{n}}|\widehat{u}(\xi)|^{2} \mu\left(|\xi|^{2}\right) d \xi\right)^{\frac{1}{2}}
\end{gathered}
$$

are equivalent (semi-) norms on $\mathcal{S}\left(\mathbb{R}^{n}\right)$.

Let us now consider the rotationally invariant case. Comparing (16) with (19) we would like to relate

$$
m(t) \quad \text { with } \quad g\left(\frac{1}{t}\right) \frac{1}{t^{n / 2}} \quad \text { and } \quad f\left(|\xi|^{2}\right) \text { with } \quad \mu\left(|\xi|^{2}\right) .
$$

Lemma 3.4. Let $g(t)=\int_{0+\frac{t}{s+t}}^{\infty} \rho(d s)$ be a complete Bernstein function. Then the functions $r \mapsto g\left(r^{-1}\right)$ and $r \mapsto r^{-n / 2} g\left(r^{-1}\right)$ are completely monotone. In particular, we have the representations

$$
\begin{aligned}
g\left(\frac{1}{r}\right) & =\int_{0}^{\infty} e^{-x r}\left(\int_{0+}^{\infty} e^{-x / s} \frac{\rho(d s)}{s}\right) d x \\
r^{-n / 2} g\left(\frac{1}{r}\right) & =\int_{0}^{\infty} e^{-x r}\left(\frac{1}{\Gamma\left(\frac{n}{2}\right)} \int_{0+}^{\infty} s^{n / 2-1} e^{-x / s} \int_{0}^{x / s} y^{n / 2-1} e^{y} d y \rho(d s)\right) d x .
\end{aligned}
$$

Proof. Since the formulae (20) and (21) prove that $r \mapsto r^{-n / 2} g\left(r^{-1}\right), n \geqslant 0$, is completely monotone, it is enough to establish these two representations.

From the definition of $g$ we have

$$
g\left(\frac{1}{r}\right)=\int_{0+}^{\infty} \frac{\frac{1}{r}}{s+\frac{1}{r}} \rho(d s)=\int_{0+}^{\infty} \frac{1}{r s+1} \rho(d s) .
$$

Since we can write

$$
\frac{1}{r s+1}=\int_{0}^{\infty} e^{-t(r s+1)} d t=\int_{0}^{\infty} e^{-x / s} e^{-x r} \frac{d x}{s}
$$

we can apply Tonelli's Theorem to find

$$
g\left(\frac{1}{r}\right)=\int_{0+}^{\infty} \int_{0}^{\infty} e^{-x / s} e^{-x r} \frac{d x}{s} \rho(d s)=\int_{0}^{\infty} e^{-x r}\left(\int_{0+}^{\infty} e^{-x / s} \frac{\rho(d s)}{s}\right) d x
$$

which proves (20). To prove (21), we use the elementary identity

$$
r^{-n / 2}=\frac{1}{\Gamma\left(\frac{n}{2}\right)} \int_{0}^{\infty} x^{n / 2-1} e^{-r x} d x, \quad n>0,
$$


and the convolution theorem for the (one-sided) Laplace transform,

$$
\mathcal{L}(u \star w)=\mathcal{L} u \cdot \mathcal{L} w,
$$

where $\mathcal{L} u(x)=\int_{0}^{\infty} e^{-x y} u(y) d y$ and

$$
u \star w(x)=\int_{0}^{x} u(x-r) w(r) d r .
$$

Setting $u(r):=\int_{0+}^{\infty} e^{-r / s} / s \rho(d s)$ and $w(r):=\Gamma\left(\frac{n}{2}\right)^{-1} r^{n / 2-1}$ we find

$$
r^{-n / 2} g\left(\frac{1}{r}\right)=\int_{0}^{\infty} e^{-r x} u \star w(x) d x .
$$

Finally,

$$
\begin{aligned}
u \star w(x) & =\int_{0}^{x} \frac{1}{\Gamma\left(\frac{n}{2}\right)} t^{n / 2-1}\left(\int_{0+}^{\infty} e^{-(x-t) / s} \frac{\rho(d s)}{s}\right) d t \\
& =\frac{1}{\Gamma\left(\frac{n}{2}\right)} \int_{0+}^{\infty} e^{-x / s}\left(\int_{0}^{x} t^{n / 2} e^{t / s} \frac{d t}{t}\right) \frac{\rho(d s)}{s} \\
& =\frac{1}{\Gamma\left(\frac{n}{2}\right)} \int_{0+}^{\infty} s^{n / 2-1} e^{-x / s}\left(\int_{0}^{x / s} y^{n / 2-1} e^{y} d y\right) \rho(d s),
\end{aligned}
$$

and (21) follows.

Lemma 3.5. Let $g:[0, \infty) \rightarrow[0, \infty)$ be a monotone increasing function with $g(0)=0$. If

$$
G:=\int_{0}^{1} g(s) \frac{d s}{s}+\int_{1}^{\infty} \frac{g(s)}{s} \frac{d s}{s}<\infty,
$$

then the function $\mu(t)$ defined in Lemma 3.3 is finitely valued and

$$
\mu(t)=\int_{0}^{t} g(s) \frac{d s}{s}+t \int_{t}^{\infty} \frac{g(s)}{s} \frac{d s}{s} .
$$

Conversely, if $\mu(1)<\infty$, then $G<\infty$.

Proof. By definition, $\mu(t):=\int_{0}^{t} \int_{r}^{\infty} g(s) / s^{2} d s d r$ so that

$$
\mu(1)=\int_{0}^{1}\left(\int_{r}^{\infty} g(s) \frac{d s}{s^{2}}\right) d r \geqslant \int_{0}^{1}\left(\int_{1}^{\infty} g(s) \frac{d s}{s^{2}}\right) d r=\int_{1}^{\infty} \frac{g(s)}{s} \frac{d s}{s}
$$

and

$$
\mu(1)=\int_{0}^{1}\left(\int_{r}^{\infty} g(s) \frac{d s}{s^{2}}\right) d r \geqslant \int_{0}^{1} g(r)\left(\int_{r}^{\infty} \frac{d s}{s^{2}}\right) d r=\int_{0}^{1} g(r) \frac{d r}{r} .
$$


Hence, $\mu(1)<\infty$ implies $G<\infty$.

Now suppose that $G<\infty$. Integration by parts yields

$$
\begin{aligned}
\mu(t) & =\int_{0}^{t}\left(\int_{r}^{\infty} g(s) \frac{d s}{s^{2}}\right) d r \\
& =\left[r \int_{r}^{\infty} g(s) \frac{d s}{s^{2}}\right]_{r=0}^{r=t}+\int_{0}^{t} s g(s) \frac{d s}{s^{2}} \\
& =t \int_{t}^{\infty} \frac{g(s)}{s} \frac{d s}{s}+\int_{0}^{t} g(s) \frac{d s}{s}-\lim _{r \rightarrow 0}\left(r \int_{r}^{\infty} \frac{g(s)}{s} \frac{d s}{s}\right) .
\end{aligned}
$$

Since $g(r t) / t \leqslant g(t) / t$ if $0 \leqslant r \leqslant 1$, and since by assumption $t \mapsto g(t) / t$ is integrable at $+\infty$, the dominated convergence theorem applies and

$$
\lim _{r \rightarrow 0}\left(r \int_{r}^{\infty} \frac{g(s)}{s} \frac{d s}{s}\right)=\lim _{r \rightarrow 0} \int_{1}^{\infty} g(r t) \frac{d t}{t}=0,
$$

where we used that $g(0)=0$.

Lemma 3.6. Let $g(r)=\int_{0+}^{\infty} \frac{r}{s+r} \rho(d s)$ be a complete Bernstein function and let $\mu(t)$ be as in Lemma 3.3. Then $\mu(1)<\infty$ - and, by Lemma 3.5, $G=$ $\int_{0}^{1} g(s) / s d s+\int_{1}^{\infty} g(s) / s^{2} d s<\infty-i f$, and only if, the measure $\rho$ representing $g$ satisfies

$$
\int_{0+}^{1} \log \left(1+s^{-1}\right) \rho(d s)+\int_{1}^{\infty} \log (1+s) \frac{\rho(d s)}{s}<\infty
$$

Proof. Because of Tonelli's Theorem we find

$$
\begin{aligned}
\int_{0}^{1} g(s) \frac{d s}{s} & =\int_{0}^{1}\left(\int_{0+}^{\infty} \frac{\rho(d r)}{s+r}\right) d s \\
& =\int_{0+}^{\infty}\left(\int_{0}^{1} \frac{d s}{s+r}\right) \rho(d r) \\
& =\left.\int_{0+}^{\infty} \log (s+r)\right|_{s=0} ^{s=1} \rho(d r) \\
& =\int_{0+}^{1} \log \left(1+r^{-1}\right) \rho(d r)+\int_{1}^{\infty} \log \left(1+r^{-1}\right) \rho(d r) .
\end{aligned}
$$

Since $\log \left(1+r^{-1}\right) \leqslant r^{-1}$ and since, by assumption, $\int_{1}^{\infty} r^{-1} \rho(d r)<\infty$ we find that

$$
\int_{0}^{1} g(s) \frac{d s}{s}<\infty \text { if, and only if, } \quad \int_{0+}^{1} \log \left(1+r^{-1}\right) \rho(d r)<\infty \text {. }
$$


Analogously, we have

$$
\begin{aligned}
\int_{1}^{\infty} \frac{g(s)}{s} \frac{d s}{s} & =\int_{1}^{\infty}\left(\int_{0+}^{\infty} \frac{\rho(d r)}{s+r}\right) \frac{d s}{s} \\
& =\int_{0+}^{1} \frac{\log (1+r)}{r} \rho(d r)+\int_{1}^{\infty} \frac{\log (1+r)}{r} \rho(d r) .
\end{aligned}
$$

Now we use that $\log (1+r) \leqslant r$, i.e., $\int_{0+}^{1} \log (1+r) / r \rho(d r) \leqslant \int_{0+}^{1} 1 \rho(d r)$ which is finite by assumption. Thus,

$$
\int_{1}^{\infty} \frac{g(s)}{s} \frac{d s}{s}<\infty \text { if, and only if, } \int_{1}^{\infty} \frac{\log (1+r)}{r} \rho(d r)<\infty .
$$

Lemma 3.7. Let $g(s)=\int_{0+}^{\infty} \frac{s}{s+r} \rho(d r)$ be a complete Bernstein function such that $G=\int_{0}^{1} g(s) / s d s+\int_{1}^{\infty} g(s) / s^{2} d s<\infty$. Then the function

$$
\mu(t)=\int_{0}^{t}\left(\int_{r}^{\infty} g(s) \frac{d s}{s^{2}}\right) d r, \quad t>0,
$$

is also a complete Bernstein function with representation

$$
\mu(t)=\int_{0+}^{\infty} \frac{t}{s+t} \nu(d s)
$$

where the measure $\nu$ is given by

$$
\nu(A)=\int_{0}^{1}\left(\int_{0}^{\infty} \rho(x y \cdot A) \frac{d y}{y^{2}}\right) \frac{d x}{x}
$$

for all Borel sets $A \subset(0, \infty)$. Alternatively,

$$
\mu(t)=\int_{0}^{\infty}\left(1-e^{-\lambda t}\right)\left(\frac{1}{\lambda^{2}} \int_{0+}^{\infty} \frac{1-e^{-\lambda z}}{z} \rho(d z)\right) d \lambda .
$$

Proof. Using the fact that all integrands are nonnegative, a straightforward calculation gives

$$
\begin{aligned}
\mu(t) & =\int_{0}^{t} \int_{r}^{\infty} g(s) \frac{d s}{s^{2}} d r \\
& =\int_{0}^{t}\left(\int_{1}^{\infty} \frac{g(r y)}{y} \frac{d y}{y}\right) \frac{d r}{r} \\
& =t \int_{0}^{1}\left(\int_{1}^{\infty} \frac{g(t x y)}{t x y} \frac{d y}{y}\right) d x \\
& =\int_{0}^{1}\left(\int_{1}^{\infty}\left[\int_{0+}^{\infty} \frac{t}{z+t x y} \rho(d z)\right] \frac{d y}{y}\right) d x \\
& =\int_{0}^{1}\left(\int_{1}^{\infty}\left[\int_{0+}^{\infty} \frac{t}{s+t} \rho(x y \cdot d s)\right] \frac{d y}{y^{2}}\right) \frac{d x}{x}
\end{aligned}
$$


and we get (23) if we define $\nu$ by (24). Since

$$
\frac{t}{s+t}=\int_{0}^{\infty}\left(1-e^{-\lambda t}\right) s e^{-\lambda s} d \lambda
$$

we find

$$
\begin{aligned}
\mu(t) & =\int_{0}^{\infty}\left(\int_{0+}^{\infty}\left(1-e^{-\lambda t}\right) s e^{-\lambda s} \nu(d s)\right) d \lambda \\
& =\int_{0}^{\infty}\left(1-e^{-\lambda t}\right)\left(\int_{0+}^{\infty} s e^{-\lambda s} \nu(d s)\right) d \lambda .
\end{aligned}
$$

Finally,

$$
\begin{aligned}
\int_{0}^{\infty} & s e^{-\lambda s} \nu(d s) \\
& =\int_{0}^{1}\left(\int_{1}^{\infty}\left[\int_{0+}^{\infty} s e^{-\lambda s} \rho(x y \cdot d s)\right] \frac{d y}{y^{2}}\right) \frac{d x}{x} \\
& =\int_{0}^{1}\left(\int_{1}^{\infty}\left[\int_{0+}^{\infty} \frac{z}{x y} \exp \left(-\frac{\lambda z}{x y}\right) \rho(d z)\right] \frac{d y}{y^{2}}\right) \frac{d x}{x} \quad(z=x y s) \\
& =\int_{0}^{1}\left(\int_{1}^{\infty}\left[\int_{0+}^{\infty} z r e^{-\lambda z \xi r} \rho(d z)\right] d \xi\right) d r \\
& =\int_{0+}^{\infty}\left(\int_{0}^{1}\left[\int_{1}^{\infty} e^{-\lambda z \xi r} d \xi\right] z r d r\right) \rho(d z) \\
& =\int_{0+}^{\infty}\left(\int_{0}^{1} \frac{e^{-\lambda z r}}{\lambda} d r\right) \rho(d z) \\
& =\frac{1}{\lambda^{2}} \int_{0+}^{\infty} \frac{1-e^{-\lambda z}}{z} \rho(d z),
\end{aligned}
$$

and the proof is finished.

\section{Equivalent Seminorms}

Let $\psi: \mathbb{R}^{n} \rightarrow \mathbb{R}$ be a c.n.d.f. of the form

$$
\psi(\xi)=\int_{y \neq 0}(1-\cos y \xi) m\left(|y|^{2}\right) d y
$$

where

$$
m(r)=\int_{0+}^{\infty} e^{-r s} \nu(d s)
$$


with a measure $\nu$ on $(0, \infty)$ such that $\int_{0+}^{1} s^{-n / 2} \nu(d s)+\int_{1}^{\infty} s^{-n / 2-1} \nu(d s)<\infty$. According to Lemma 2.1 we have $\psi(\xi)=f\left(|\xi|^{2}\right)$ where $f$ is given by (15). Moreover we know, cf. (16), that

$$
\int_{\mathbb{R}^{n}}|\widehat{u}(\xi)|^{2} f\left(|\xi|^{2}\right) d \xi=\frac{1}{2} \iint_{\mathbb{R}^{n} \times \mathbb{R}^{n}}|u(x+y)-u(x)|^{2} m\left(|y|^{2}\right) d y d x
$$

holds. On the other hand, for a completely monotone function $g$ we know from Lemma 3.3 that

$$
\int_{\mathbb{R}^{n}}|\widehat{u}(\xi)|^{2} \mu\left(|\xi|^{2}\right) d \xi \sim \iint_{\mathbb{R}^{n} \times \mathbb{R}^{n}}|u(x+y)-u(x)|^{2} g\left(\frac{1}{|y|^{2}}\right) \frac{d x d y}{|y|^{n}}
$$

in the sense of equivalent (semi-)norms where $\mu(t)$ is as in Lemma 3.3, i.e.,

$$
\mu(t)=\int_{0}^{t}\left(\int_{r}^{\infty} g(s) \frac{d s}{s^{2}}\right) d r
$$

Both $m(r)$ and $r \mapsto r^{-n / 2} g\left(\frac{1}{r}\right)$ are completely monotone functions. Our first aim is to compare these two functions. From Lemma 3.4 we know that

$$
r^{-n / 2} g\left(\frac{1}{r}\right)=\int_{0}^{\infty} e^{-x r} h(x) d x
$$

where

$$
h(x)=\frac{x^{n / 2}}{\Gamma\left(\frac{n}{2}\right)} \int_{0+}^{\infty} e^{-x / s}\left(\int_{0}^{1} t^{n / 2} e^{t x / s} \frac{d t}{t}\right) \frac{\rho(d s)}{s} .
$$

Lemma 4.1. If $\mu(1)<\infty$, then $\int_{0}^{1} x^{-n / 2} h(x) d x+\int_{1}^{\infty} x^{-n / 2-1} h(x) d x<\infty$.

Proof. Assume that $\mu(1)<\infty$. Then

$$
\begin{aligned}
\int_{0}^{1} x^{-n / 2} h(x) d x & =\int_{0}^{1} x^{-n / 2} \frac{x^{n / 2}}{\Gamma\left(\frac{n}{2}\right)}\left(\int_{0+}^{\infty} e^{-x / s}\left[\int_{0}^{1} t^{n / 2} e^{t x / s} \frac{d t}{t}\right] \frac{\rho(d s)}{s}\right) d x \\
& =\frac{1}{\Gamma\left(\frac{n}{2}\right)} \int_{0}^{1}\left(\int_{0+}^{\infty}\left[\int_{0}^{1} e^{-x(1-t) / s} t^{n / 2-1} d t\right] \frac{\rho(d s)}{s}\right) d x \\
& =\frac{1}{\Gamma\left(\frac{n}{2}\right)} \int_{0+}^{\infty}\left(\int_{0}^{1}\left[\frac{-s}{1-t} e^{-x(1-t) / s}\right]_{x=0}^{x=1} t^{n / 2-1} d t\right) \frac{\rho(d s)}{s} \\
& =\frac{1}{\Gamma\left(\frac{n}{2}\right)} \int_{0+}^{\infty}\left(\int_{0}^{1} \frac{1}{1-t}\left(1-e^{-(1-t) / s}\right) t^{n / 2-1} d t\right) \rho(d s)
\end{aligned}
$$


Since $1-e^{-\lambda} \leqslant \lambda \wedge 1 \leqslant 2 \lambda /(1+\lambda)$ for all $\lambda>0$, we find

$$
\begin{aligned}
\int_{0}^{1} x^{-n / 2} h(x) d x & \leqslant \frac{2}{\Gamma\left(\frac{n}{2}\right)} \int_{0+}^{\infty}\left(\int_{0}^{1} \frac{1}{1-t} \frac{\frac{1-t}{s}}{1+\frac{1-t}{s}} t^{n / 2-1} d t\right) \rho(d s) \\
& =\frac{2}{\Gamma\left(\frac{n}{2}\right)} \int_{0}^{1}\left(\int_{0+}^{\infty} \frac{1}{s+1-t} \rho(d s)\right) t^{n / 2-1} d t \\
& =\frac{2}{\Gamma\left(\frac{n}{2}\right)} \int_{0}^{1} \frac{g(1-t)}{1-t} t^{n / 2-1} d t \\
& =\frac{2}{\Gamma\left(\frac{n}{2}\right)}\left(\int_{0}^{1 / 2} \frac{g(1-t)}{1-t} t^{n / 2-1} d t+\int_{1 / 2}^{1} \frac{g(1-t)}{1-t} t^{n / 2-1} d t\right) \\
& \leqslant c_{n}^{\prime}\left(\int_{0}^{1 / 2} \frac{g\left(\frac{1}{2}\right)}{\frac{1}{2}} t^{n / 2-1} d t+\int_{1 / 2}^{1} \frac{g(1-t)}{1-t} t^{n / 2-1} d t\right)
\end{aligned}
$$

where we use that $s \mapsto g(s) / s$ is decreasing. Thus,

$$
\begin{aligned}
\int_{0}^{1} x^{-n / 2} h(x) d x & \leqslant \tilde{c}_{n}\left(\int_{0}^{1 / 2} t^{n / 2-1} d t+\int_{1 / 2}^{1} \frac{g(1-t)}{1-t} t^{n / 2-1} d t\right) \\
& \leqslant c_{n}\left(\int_{0}^{1 / 2} t^{n / 2-1} d t+\int_{0}^{1} \frac{g(s)}{s} d s\right)<\infty
\end{aligned}
$$

To show the finiteness of the second integral in the statement, we use the elementary estimate $e^{-\lambda} \leqslant 1 /(1+\lambda), \lambda \geqslant 0$, to get

$$
\begin{aligned}
\int_{1}^{\infty} & x^{-n / 2-1} h(x) d x \\
& =\int_{1}^{\infty} x^{-n / 2-1} \frac{x^{n / 2}}{\Gamma\left(\frac{n}{2}\right)}\left(\int_{0+}^{\infty} e^{-x / s}\left[\int_{0}^{1} t^{n / 2} e^{t x / s} \frac{d t}{t}\right] \frac{\rho(d s)}{s}\right) d x \\
& =\frac{1}{\Gamma\left(\frac{n}{2}\right)} \int_{1}^{\infty}\left(\int_{0}^{1}\left[\int_{0+}^{\infty} e^{-x(1-t) / s} t^{n / 2-1} \frac{\rho(d s)}{s}\right] d t\right) \frac{d x}{x} \\
& \leqslant \frac{1}{\Gamma\left(\frac{n}{2}\right)} \int_{1}^{\infty}\left(\int_{0}^{1}\left[\int_{0+}^{\infty} \frac{1}{1+\frac{x(1-t)}{s}} \frac{1}{s} t^{n / 2-1} \rho(d s)\right] d t\right) \frac{d x}{x} \\
& =\frac{1}{\Gamma\left(\frac{n}{2}\right)} \int_{1}^{\infty}\left(\int_{0}^{1}\left[\int_{0+}^{\infty} \frac{1}{s+x(1-t)} \rho(d s)\right] t^{n / 2-1} d t\right) \frac{d x}{x} \\
& =\frac{1}{\Gamma\left(\frac{n}{2}\right)} \int_{1}^{\infty}\left(\int_{0}^{1} \frac{g(x(1-t))}{x(1-t)} t^{n / 2-1} d t\right) \frac{d x}{x} .
\end{aligned}
$$


Again we use that $s \mapsto g(s) / s$ is decreasing to find

$$
\begin{aligned}
\int_{1}^{\infty} & x^{-n / 2-1} h(x) d x \\
& \leqslant c_{n}^{\prime}\left\{\int_{1}^{\infty}\left[\int_{0}^{\frac{1}{2}} \frac{g\left(\frac{x}{2}\right)}{\frac{x}{2}} t^{n / 2-1} d t\right] \frac{d x}{x}+\int_{1}^{\infty}\left[\int_{\frac{1}{2}}^{1} \frac{g\left(\frac{x}{2}\right)}{\frac{x}{2}} d t\right] \frac{d x}{x}\right\} \\
& \leqslant \tilde{c}_{n}\left\{\left(\int_{1}^{\infty} \frac{g\left(\frac{x}{2}\right)}{\left(\frac{x}{2}\right)^{2}} d x\right)\left(\int_{0}^{\frac{1}{2}} t^{n / 2-1} d t\right)+\int_{1}^{\infty}\left(\int_{0}^{\frac{1}{2}} \frac{g(x s)}{x s} d s\right) \frac{d x}{x}\right\} .
\end{aligned}
$$

By assumption, the first term is finite, and for the second term we find

$$
\int_{1}^{\infty}\left(\int_{0}^{\frac{1}{2}} \frac{g(x s)}{x s} d s\right) \frac{d x}{x}=\int_{0}^{\frac{1}{2}}\left(\int_{s}^{\infty} \frac{g(y)}{y} \frac{d y}{y}\right) d s=\mu\left(\frac{1}{2}\right)<\infty
$$

as $\mu\left(\frac{1}{2}\right) \leqslant \mu(1)<\infty$.

Corollary 4.2. The Bernstein function $f$ associated with $s \mapsto g\left(s^{-1}\right) s^{-n / 2}$ is given by

$$
f(r)=\frac{1}{4} \frac{\pi^{n / 2}}{\Gamma\left(\frac{n}{2}\right)} \int_{0}^{\infty} \frac{1-e^{-r \lambda}}{\lambda^{2}}\left(\int_{0+}^{\infty}\left[\int_{0}^{1} \exp \left(-\frac{1-t}{4 s \lambda}\right) t^{n / 2-1} d t\right] \frac{\rho(d s)}{s}\right) d \lambda .
$$

Proof. Applying Lemma 2.1 to the measure $\eta(d x)=h(x) d x, x>0$, we find

$$
f(r)=\int_{0+}^{\infty}\left(1-e^{-r x}\right)(4 \pi x)^{n / 2} \Phi(\eta)(d x)
$$

where $\Phi(x)=1 / 4 x$. Hence

$$
\begin{aligned}
f(r) & =\frac{\pi^{n / 2}}{\Gamma\left(\frac{n}{2}\right)} \int_{0}^{\infty}\left(1-e^{-r / 4 x}\right) x^{-n / 2} x^{n / 2}\left(\int_{0+}^{\infty} e^{-x / s}\left[\int_{0}^{1} t^{n / 2-1} e^{-t x / s} d t\right] \frac{\rho(d s)}{s}\right) d x \\
& =\frac{\pi^{n / 2}}{\Gamma\left(\frac{n}{2}\right)} \int_{0}^{\infty}\left(\int_{0+}^{\infty}\left[\int_{0}^{1}\left(1-e^{-r / 4 x}\right) e^{-x(1-t) / s} t^{n / 2-1} d t\right] \frac{\rho(d s)}{s}\right) d x \\
& =\frac{\pi^{n / 2}}{4 \Gamma\left(\frac{n}{2}\right)} \int_{0}^{\infty}\left(\int_{0+}^{\infty}\left[\int_{0}^{1}\left(1-e^{-r \lambda}\right) \exp \left(-\frac{1-t}{4 s \lambda}\right) t^{n / 2-1} d t\right] \frac{\rho(d s)}{s}\right) \frac{d \lambda}{\lambda^{2}},
\end{aligned}
$$

where we used the substitution $\lambda=1 / 4 x$.

It is by no means obvious to find out the cases where $f$ and $\mu$ are equal. However, we can always prove that $f \sim \mu$ holds.

Proposition 4.3. Assume that $f$ is as in the above corollary, $\mu$ as in Lemma 3.3 and that the assumptions of Lemma 4.1 are satisfied. Then there are two constants $\kappa_{0}, \kappa_{1}>0$ such that

$$
\kappa_{0} f(r) \leqslant \mu(r) \leqslant \kappa_{1} f(r), \quad r>0 .
$$


Proof. We begin with the elementary remark that $1-e^{-x} \sim x /(1+x)$ for all $x \geqslant 0$. Therefore, we find

$$
\begin{aligned}
f(r) & =\frac{\pi^{n / 2}}{4 \Gamma\left(\frac{n}{2}\right)} \int_{0}^{\infty} \frac{1-e^{-r \lambda}}{\lambda^{2}}\left(\int_{0+}^{\infty}\left[\int_{0}^{1} \exp \left(-\frac{1-t}{4 s \lambda}\right) t^{n / 2-1} d t\right] \frac{\rho(d s)}{s}\right) d \lambda . \\
& \sim \int_{0}^{\infty}\left(\int_{0+}^{\infty}\left[\int_{0}^{1} \frac{r \lambda}{1+r \lambda} \exp \left(-\frac{1-t}{4 s \lambda}\right) t^{n / 2-1} d t\right] \frac{\rho(d s)}{s}\right) d \lambda \\
& =\int_{0}^{\infty}\left(\int_{0+}^{\infty}\left[\int_{0}^{1} \frac{r \frac{x}{s}}{1+r \frac{x}{s}} \exp \left(-\frac{1-t}{4 x}\right) t^{n / 2-1} d t\right] \rho(d s)\right) \frac{d x}{x^{2}} \\
& =\int_{0}^{\infty}\left(\int_{0}^{1}\left[\int_{0+}^{\infty} \frac{r x}{s+r x} \rho(d s)\right] \exp \left(-\frac{1-t}{4 x}\right) t^{n / 2-1} d t\right) \frac{d x}{x^{2}} \\
& =\left\{\int_{0}^{1}+\int_{1}^{\infty}\right\}\left(\int_{0}^{1} g(r x) \exp \left(-\frac{1-t}{4 x}\right) t^{n / 2-1} d t\right) \frac{d x}{x^{2}} \\
& =: I_{1}+I_{2} .
\end{aligned}
$$

We will estimate $I_{1}$ and $I_{2}$ separately.

$$
\begin{aligned}
I_{2} & =\int_{r}^{\infty}\left(\int_{0}^{1} g(y) \exp \left(-\frac{(1-t) r}{4 y}\right) t^{n / 2-1} d t\right) \frac{r d y}{y^{2}} \\
& \leqslant r \int_{r}^{\infty} g(y) \frac{d y}{y^{2}} \int_{0}^{1} t^{n / 2-1} d t \\
& \leqslant c_{1} r \int_{r}^{\infty} g(y) \frac{d y}{y^{2}} .
\end{aligned}
$$

On the other hand, we find

$$
\begin{aligned}
I_{2} & =\int_{r}^{\infty}\left(\int_{0}^{1} g(y) \exp \left(-\frac{(1-t) r}{4 y}\right) t^{n / 2-1} d t\right) \frac{r d y}{y^{2}} \\
& \geqslant r \int_{r}^{\infty}\left(\int_{0}^{1} g(y) e^{-1 / 4} t^{n / 2-1} d t\right) \frac{d y}{y^{2}} \\
& \geqslant c_{2} r \int_{r}^{\infty} g(y) \frac{d y}{y^{2}}
\end{aligned}
$$

so that

$$
I_{2} \sim r \int_{r}^{\infty} g(y) \frac{d y}{y^{2}} .
$$

Let us now estimate $I_{1}$. We have

$$
\begin{aligned}
I_{1} & =\int_{0}^{1}\left(\int_{0}^{r} g(y) \exp \left(-\frac{(1-t) r}{4 y}\right) t^{n / 2-1} r \frac{d y}{y^{2}}\right) d t \\
& =4 \int_{0}^{r}\left(\int_{0}^{1} \frac{r}{4 y} \exp \left(-\frac{(1-t) r}{4 y}\right) t^{n / 2-1} d t\right) g(y) \frac{d y}{y} .
\end{aligned}
$$


Setting

we see

$$
J(\lambda):=\int_{0}^{1} \lambda e^{-(1-t) \lambda} t^{n / 2-1} d t \quad \text { and } \quad \lambda:=\frac{r}{4 y}
$$

$$
I_{1}=4 J(\lambda) \int_{0}^{r} g(y) \frac{d y}{y}
$$

and it remains to show that $0<c \leqslant J(\lambda) \leqslant C<\infty$ for all $\lambda \geqslant \frac{1}{4}$. This will be done in Lemma 4.4 below. From this we conclude that

$$
I_{1} \sim \int_{0}^{r} g(y) \frac{d y}{y} .
$$

From Lemma 3.5 we know that

$$
\mu(r)=\int_{0}^{r} g(y) \frac{d y}{y}+r \int_{r}^{\infty} g(y) \frac{d y}{y^{2}},
$$

and collecting all of the above calculations gives

$$
\mu(r) \sim I_{1}+I_{2} \sim f(r) .
$$

Lemma 4.4. The function

$$
J(\lambda):=\lambda \int_{0}^{1} e^{-(1-t) \lambda} t^{n / 2-1} d t, \quad \lambda>0,
$$

satisfies $0<\min \left\{1-e^{1 / 4}, J\left(\frac{1}{4}\right)\right\} \leqslant J(\lambda) \leqslant 1$ for all $\lambda \geqslant \frac{1}{4}$.

Proof. For $n \geqslant 2$ we have

$$
J(\lambda) \leqslant \lambda \int_{0}^{1} e^{-(1-t) \lambda} d t=1-e^{-\lambda} \leqslant 1,
$$

and for $n=1$ we set $s=t / \lambda$ to find

$$
J(\lambda)=\sqrt{\lambda} e^{-\lambda} \int_{0}^{\lambda} e^{-s} s^{1 / 2-1} d s \leqslant \sqrt{\lambda} e^{-\lambda} \int_{0}^{\infty} e^{-s} s^{1 / 2-1} d s=\sqrt{\lambda} e^{-\lambda} \Gamma\left(\frac{1}{2}\right) .
$$

Clearly, $\sqrt{\lambda} e^{-\lambda} \Gamma\left(\frac{1}{2}\right)=\sqrt{\pi \lambda} e^{-\lambda}<1$.

To get the lower bounds for $n=1,2$ observe that $0 \leqslant t \leqslant 1$ implies that $t^{n / 2-1} \geqslant 1$, and so

$$
J(\lambda) \geqslant \lambda \int_{0}^{1} e^{-(1-t) \lambda} d t=1-e^{-\lambda} \geqslant 1-e^{-1 / 4} .
$$

For $n \geqslant 3$ we have with $a:=\frac{n}{2}-1>0$

$$
J(\lambda)=\int_{0}^{\lambda} e^{-t}\left(1-\frac{t}{\lambda}\right)^{a} d t
$$


and so

$$
J^{\prime}(\lambda)=\frac{a}{\lambda^{2}} \int_{0}^{\lambda} e^{-t}\left(1-\frac{t}{\lambda}\right)^{a-1} d t \geqslant 0,
$$

implying that $\lambda \mapsto J(\lambda)$ is monotone increasing. Hence, $J\left(\frac{1}{4}\right) \leqslant J(\lambda)$ for all $\lambda \geqslant \frac{1}{4}$ which finally proves the lemma.

We can now formulate our main result.

Theorem 4.5. Let $g(t)=\int_{0+\frac{t}{s+t}}^{\infty} \rho(d s)$ be a complete Bernstein function and define $\mu(t)$ by

$$
\mu(t):=\int_{0}^{t}\left(\int_{r}^{\infty} g(s) \frac{d s}{s^{2}}\right) d r .
$$

If $\mu(1)<\infty$, then

$$
f(r)=\frac{\pi^{n / 2}}{4 \Gamma\left(\frac{n}{2}\right)} \int_{0}^{\infty} \frac{1-e^{-r \lambda}}{\lambda^{2}}\left[\int_{0+}^{\infty}\left(\int_{0}^{1} \exp \left(-\frac{1-t}{4 \lambda s}\right) t^{n / 2-1} d t\right) \frac{\rho(d s)}{s}\right] d \lambda
$$

is a Bernstein function and the subordinate negative definite function

$$
\psi(\xi)=f\left(|\xi|^{2}\right)=\int_{y \neq 0}(1-\cos y \xi) m\left(|y|^{2}\right) d y
$$

satisfies the following norm-equivalence

$$
\begin{aligned}
& \frac{1}{2} \iint_{\mathbb{R}^{n} \times \mathbb{R}^{n}}|u(x+y)-u(x)|^{2} m\left(|y|^{2}\right) d y d x=\int_{\mathbb{R}^{n}}|\widehat{u}(\xi)|^{2} f\left(|\xi|^{2}\right) d \xi \\
\sim & \int_{\mathbb{R}^{n}}|\widehat{u}(\xi)|^{2} \mu\left(|\xi|^{2}\right) d \xi=\iint_{\mathbb{R}^{n} \times \mathbb{R}^{n}}|u(x+y)-u(x)|^{2} g\left(\frac{1}{|y|^{2}}\right) \frac{d y}{|y|^{n}} d x .
\end{aligned}
$$

Here we may take $u$ from $C_{c}^{\infty}\left(\mathbb{R}^{n}\right)$ or $\mathcal{S}\left(\mathbb{R}^{n}\right)$ or $H^{\psi, 1}\left(\mathbb{R}^{n}\right)$.

Remark 4.6. If $\psi_{1}: \mathbb{R}^{n} \rightarrow \mathbb{R}$ and $\psi_{2}: \mathbb{R}^{m} \rightarrow \mathbb{R}$ are two c.n.d.fs, then $(\xi, \eta) \mapsto$ $\psi_{1}(\xi)+\psi_{2}(\eta)$ is a continuous negative function on $\mathbb{R}^{n} \times \mathbb{R}^{m}$. If $f_{1}, f_{2}$ are two (complete) Bernstein functions, then $(\xi, \eta) \mapsto f_{1}\left(\psi_{1}(\xi)\right)+f_{2}\left(\psi_{2}(\eta)\right)$ is again a continuous negative definite function on $\mathbb{R}^{n} \times \mathbb{R}^{m}$. This observation is sufficient to generalise Theorem 4.5 to many anisotropic cases. In particular, the case treated by Maz'ya and Nagel [18] - see Lemma 3.2 - is included.

Remark 4.7. Since for every c.n.d.f. $\psi: \mathbb{R}^{n} \rightarrow \mathbb{R}$ the estimates

$$
1 \leqslant 1+\psi(\xi) \leqslant 2 \sup _{|\eta| \leqslant 1} \psi(\eta)\left(1+|\xi|^{2}\right), \quad \xi \in \mathbb{R}^{n},
$$

hold, we have the following continuous embeddings: $L^{2}\left(\mathbb{R}^{n}\right) \hookrightarrow H^{\psi, 1}\left(\mathbb{R}^{n}\right) \hookrightarrow$ $H^{1}\left(\mathbb{R}^{n}\right)$. However, it is not clear how the spaces $H^{\psi, 1}$ relate to the anisotropic Triebel-Lizorkin scales, cf. Triebel [25] for their definition and properties. Recently, some progress has been achieved by W. Farkas and H.-G. Leopold [7] who study scales of anisotropic Besov and Triebel-Lizorkin spaces related to negative definite functions of the form $f\left(|\xi|^{2}\right)$ for certain classes of complete Bernstein functions. 


\section{Appendix 1:}

\section{A Table of (Complete) Bernstein Functions}

Rather than giving examples of (complete) Bernstein functions in the text we decided to compile a table of Bernstein functions and their various representing measures as appendix. Where appropriate, we refer to the literature for proofs and references, otherwise we trust that the reader will be able to perform some standard calculations by himself.

Notation. The standard form of a Bernstein function is

$$
f(x)=a+b x+\int_{0+}^{\infty}\left(1-e^{-s x}\right) \tau(d s)
$$

where $a, b \geqslant 0$ and $\tau$ is a measure on $(0, \infty)$ with $\int_{0}^{\infty}(s \wedge 1) \tau(d s)<\infty$. The standard form of a complete Bernstein function is

$$
f(x)=a+b x+\int_{0+}^{\infty} \frac{x}{t+x} \rho(d t)
$$

where $a, b \geqslant 0$ and $\rho$ is a measure on $(0, \infty)$ with $\int_{0+}^{\infty}(1+t)^{-1} \rho(d t)<\infty$. Note that for a complete Bernstein function $f(x)$ the function $f(x) / x$,

$$
\frac{f(x)}{x}=\frac{a}{x}+b+\underbrace{\int_{(0, \infty)} \frac{\rho(d t)}{t+x}}_{\text {Stieltjes transform }}=\int_{[0, \infty]} \frac{\tilde{\rho}(d t)}{t+x}
$$

is a Stieltjes function (cf. Berg-Forst [4]) resp. Stieltjes transform of the measure $\tilde{\rho}(d t):=a \delta_{0}(d t)+\rho(d t)+b t \delta_{\infty}(d t)$ on $[0, \infty]$.

The interplay between complete Bernstein and Stieltjes functions is, e.g., discussed in [22]. Here we only need that a Bernstein function $f$ is a complete Bernstein function if, and only if,

$$
\tau(d s)=m(s) d s \quad \text { where } \quad m(s)=\int_{0+}^{\infty} e^{-s t} t \rho(d t) .
$$

In the table below we consider only (complete) Bernstein functions where $a=b=0$. We write $J_{\nu}(x), Y_{\nu}(x)$ for the Bessel functions of the first and second kind, $I_{\nu}(x), K_{\nu}(x)$ for the modified Bessel functions of the first and second kind and $0<j_{\nu, 1}<j_{\nu, 2}<\ldots<j_{\nu, n}<\ldots$ for the positive zeros of the Bessel function $J_{\nu}(x)$ (see, e.g., Andrews et al. [2]). 


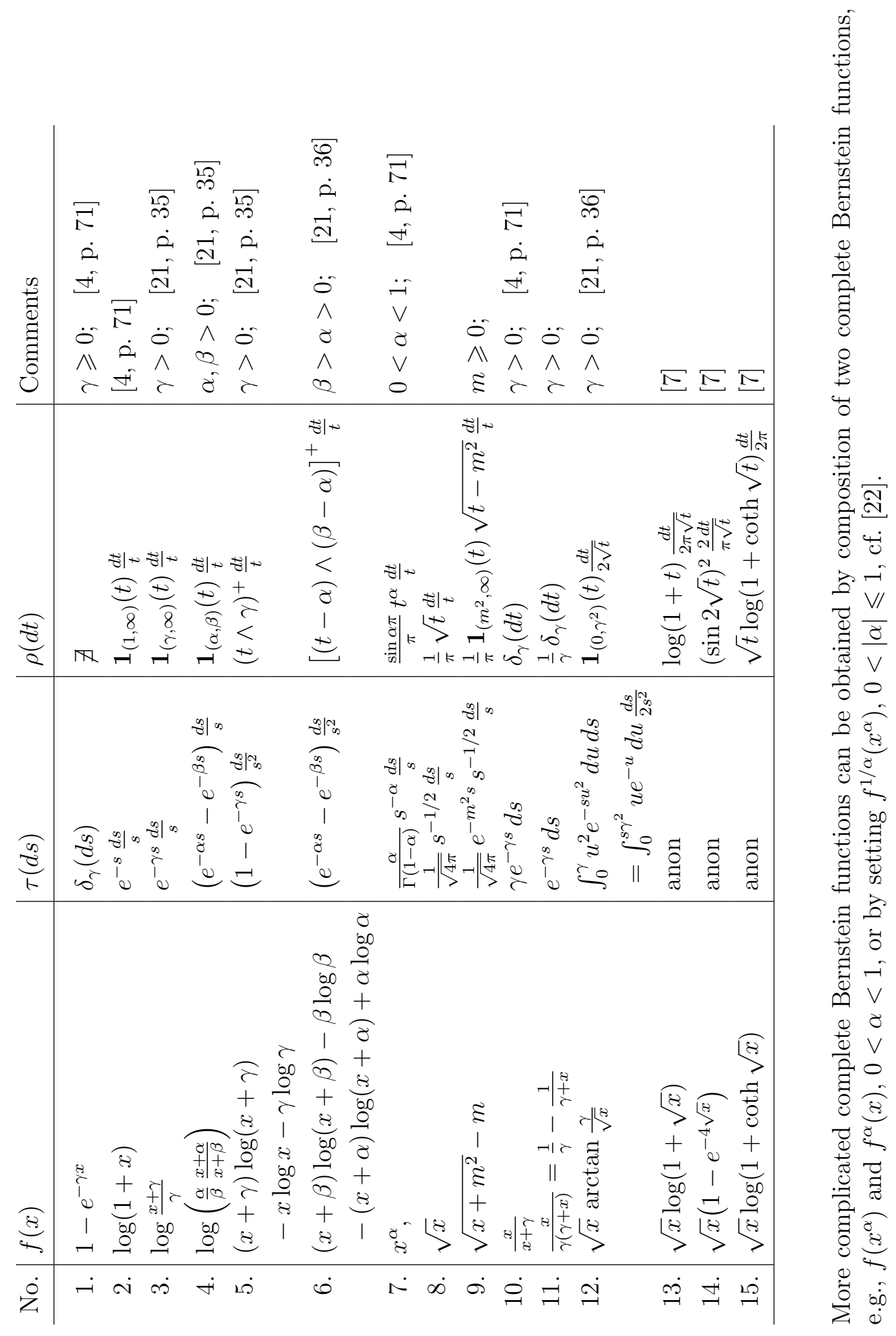




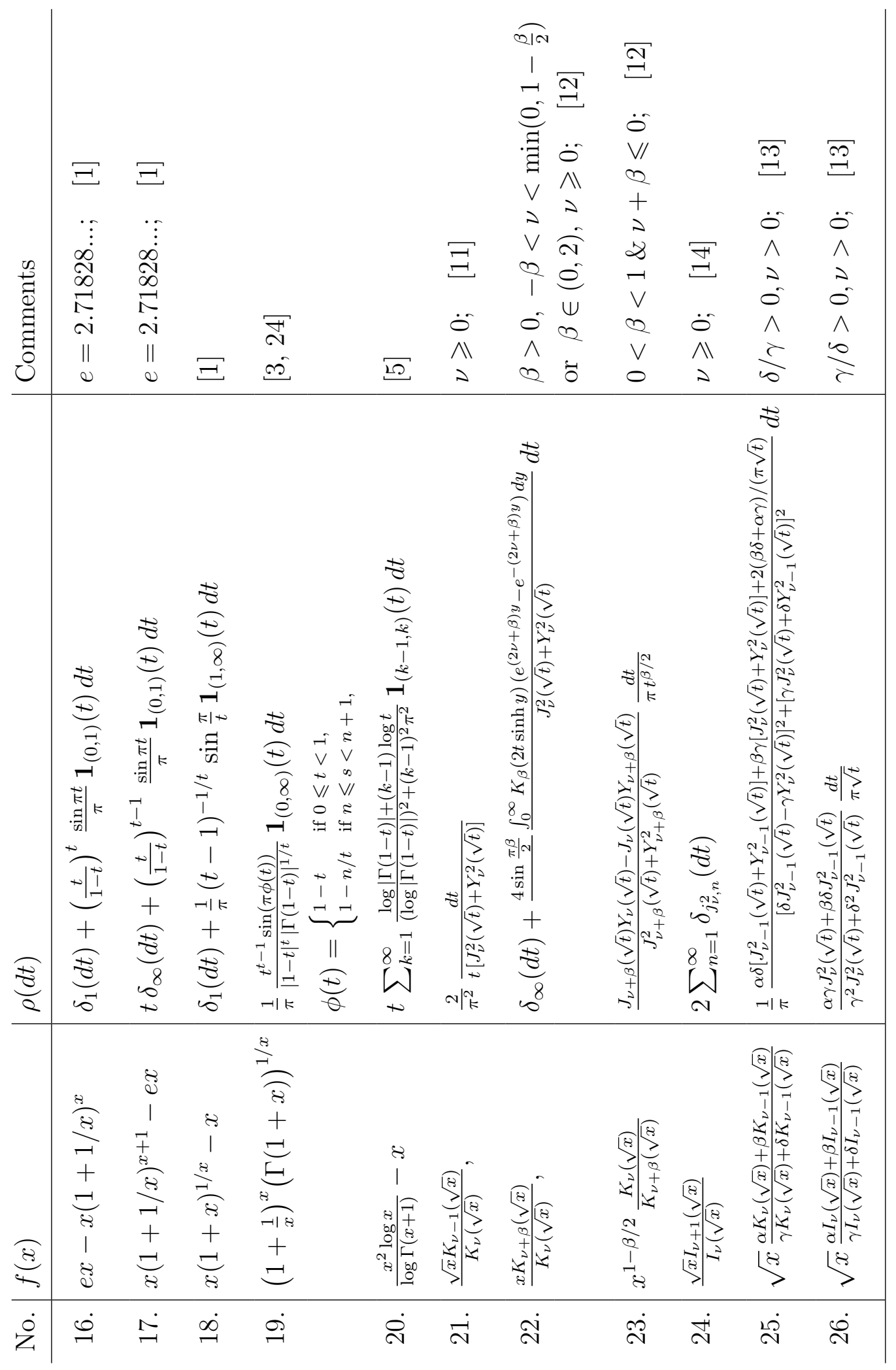


The following functions are Bernstein functions. Although they are also Stieltjes transforms, it is not clear whether they are complete Bernstein functions, since the representing measure $\rho$ (shown in the table below) might become negative.

\begin{tabular}{|c|c|c|c|}
\hline No. & $f(x)$ & $\rho(d t)$ & Comments \\
\hline 27. & $\frac{x K_{\nu}(\alpha \sqrt{x})}{K_{\nu}(\beta \sqrt{x})}$ & $\frac{J_{\nu}(\beta \sqrt{t}) Y_{\nu}(\alpha \sqrt{t})-J_{\nu}(\alpha \sqrt{t}) Y_{\nu}(\beta \sqrt{t})}{J_{\nu}^{2}(\beta \sqrt{t})+Y_{\nu}^{2}(\beta \sqrt{t})} \frac{d t}{\pi}$ & $\begin{array}{l}\alpha>\beta>0 \\
\nu \geqslant 0 ; \quad[13]\end{array}$ \\
\hline 28. & $\frac{x I_{\nu}(\beta \sqrt{x})}{I_{\nu}(\alpha \sqrt{x})}$ & $\frac{2}{\alpha^{2}} \sum_{n=1}^{\infty} \frac{j_{\nu, n} J_{\nu}\left(\beta j_{\nu, n} / \alpha\right)}{J_{\nu+1}\left(j_{\nu, n}\right)} \delta_{j_{\nu, n}^{2} / \alpha^{2}}(d t)$ & $\begin{array}{l}\alpha>\beta>0, \\
\nu>-1 ; \quad[13]\end{array}$ \\
\hline 29. & $\frac{K_{\nu}(\alpha \sqrt{x})}{K_{\nu}(\beta \sqrt{x})}-\left(\frac{\beta}{\alpha}\right)^{\nu}$ & $\frac{J_{\nu}(\alpha \sqrt{t}) Y_{\nu}(\beta \sqrt{t})-J_{\nu}(\beta \sqrt{t}) Y_{\nu}(\alpha \sqrt{t})}{J_{\nu}^{2}(\beta \sqrt{t})+Y_{\nu}^{2}(\beta \sqrt{t})} \frac{d t}{\pi t}$ & $\begin{array}{l}\alpha>\beta>0 \\
\nu \geqslant 0 ; \quad[12]\end{array}$ \\
\hline
\end{tabular}

\section{Appendix 2: \\ Proofs of Lemma 2.1 and Theorem 2.2}

We begin with the proof of Lemma 2.1.

Proof of Lemma 2.1. Assume first that $\psi(\xi)=g\left(|\xi|^{2}\right)$ with some Bernstein function $g$,

$$
g(x)=a+b x+\int_{0+}^{\infty}\left(1-e^{-x s}\right) \tau(d s) .
$$

Then

$$
\begin{aligned}
g\left(|\xi|^{2}\right) & =a+b|\xi|^{2}+\int_{0+}^{\infty}\left(1-e^{-s|\xi|^{2}}\right) \tau(d s) \\
& =a+b|\xi|^{2}+\int_{0+}^{\infty}\left(\int_{\mathbb{R}^{n}}\left(1-e^{-i y \xi}\right) \frac{1}{(4 \pi s)^{n / 2}} \exp \left(-\frac{|y|^{2}}{4 s}\right) d y\right) \tau(d s) \\
& =a+b|\xi|^{2}+\int_{\mathbb{R}^{n}}\left(1-e^{-i y \xi}\right)\left(\int_{0+}^{\infty} \frac{1}{(4 \pi s)^{n / 2}} \exp \left(-\frac{|y|^{2}}{4 s}\right) \tau(d s)\right) d y
\end{aligned}
$$

Switching to polar coordinates we see

$$
\begin{aligned}
\int_{\mathbb{R}^{n}} & \frac{|y|^{2}}{1+|y|^{2}}\left(\int_{0+}^{\infty} \frac{1}{(4 \pi s)^{n / 2}} \exp \left(-\frac{|y|^{2}}{4 s}\right) \tau(d s)\right) d y \\
\quad & \frac{2 \pi^{n / 2}}{\Gamma\left(\frac{n}{2}\right)} \int_{0+}^{\infty} \frac{r^{2}}{1+r^{2}}\left(\int_{0+}^{\infty} \frac{1}{(4 \pi s)^{n / 2}} \exp \left(-\frac{r^{2}}{4 s}\right) \tau(d s)\right) r^{n-1} d r
\end{aligned}
$$

${ }^{\ddagger}$ Based on an unpublished manuscript of the second author, see also [21]. 


$$
\begin{aligned}
& =\frac{2 \cdot 4^{-n / 2}}{\Gamma\left(\frac{n}{2}\right)} \int_{0+}^{\infty}\left(\int_{0+}^{\infty} \frac{s t^{2}}{1+s t^{2}} \tau(d s)\right) \exp \left(-\frac{t^{2}}{4}\right) t^{n-1} d t \\
& \leqslant \frac{2 \cdot 4^{-n / 2}}{\Gamma\left(\frac{n}{2}\right)}\left(\int_{0+}^{\infty} \frac{s}{1+s} \tau(d s)\right)\left(\int_{0+}^{1} t^{n-1} e^{-t^{2} / 4} d t+\int_{1}^{\infty} t^{n+1} e^{-t^{2} / 4} d t\right) \\
& <\infty
\end{aligned}
$$

Therefore, the function

$$
m\left(|y|^{2}\right):=\int_{0+}^{\infty} \exp \left(-\frac{|y|^{2}}{4 s}\right) \frac{\tau(d s)}{(4 \pi s)^{n / 2}}=\frac{1}{\pi^{n / 2}} \int_{0+}^{\infty} e^{-s|y|^{2}} s^{n / 2} \Phi(\tau)(d s)
$$

is the Laplace transform of the measure $s^{n / 2} \Phi(\tau)(d s)$ on $(0, \infty)$ where $\Phi: s \mapsto$ $(4 s)^{-1}$. Moreover, the above calculation shows that $m\left(|y|^{2}\right) d y$ has the integrability properties of a Lévy measure. Since $\tau$ is the representing measure of the Bernstein function $g$, we find

$$
\begin{gathered}
\int_{0+}^{1} s^{-n / 2} s^{n / 2} \Phi(\tau)(d s)+\int_{1}^{\infty} s^{-n / 2-1} s^{n / 2} \Phi(\tau)(d s) \\
=\int_{0+}^{1 / 4} 4 s \tau(d s)+\int_{1 / 4}^{\infty} \tau(d s)<\infty
\end{gathered}
$$

Conversely, assume that $\psi$ is of the form (14) with Lévy measure $m\left(|y|^{2}\right) d y$ where $m(r)$ is the Laplace transform of a measure $\nu$ on $(0, \infty)$ such that $\int_{0+}^{1} s^{-n / 2} \nu(d s)+\int_{1}^{\infty} s^{-n / 2-1} \nu(d s)<\infty$. Using calculations similar to the ones used above it is enough to check that the function given by (15) is indeed a Bernstein function. This, however, follows from

$$
\begin{aligned}
\int_{0+}^{\infty} \frac{s}{1+s} & s^{n / 2} \Phi(\nu)(d s)=\int_{0+}^{\infty} \frac{1}{1+4 s}(4 s)^{-n / 2} \nu(d s) \\
& \leqslant\left(\int_{0+}^{1}(4 s)^{-n / 2} \nu(d s)+\int_{1}^{\infty}(4 s)^{-n / 2-1} \nu(d s)\right)<\infty .
\end{aligned}
$$

The proof of Theorem 2.2 uses similar techniques to the ones used by Harzallah [8] to prove that the Bernstein functions are the only class of functions with the property that $f \circ \psi$ is a c.n.d.f. for all continuous negative definite functions $\psi$.

Lemma. Denote by $\mathcal{O}$ the family of functions $f:[0, \infty) \rightarrow \mathbb{R}$ such that $f\left(|\xi|^{2}\right)$ is a c.n.d.f. on every $\mathbb{R}^{n}, n \in \mathbb{N}$. Then

(a) $\mathcal{O}$ is closed under locally uniform convergence, and for all $f, g \in \mathcal{O}$

(b) $f \geqslant 0$ 
(c) $\quad \lambda f+(1-\lambda) g \in \mathcal{O}$ for all $0 \leqslant \lambda \leqslant 1$

(d) $\left.f\right|_{(0, \infty)}$ is continuous

(e) $\quad \tau_{c} f:=f\left(\bullet+c^{2}\right)-f\left(c^{2}\right) \in \mathcal{O}$ for all $c \in \mathbb{R}$

(f) $f-\tau_{c} f \in \mathcal{O}$ for all $c \in \mathbb{R}$

(g) $f$ is increasing, subadditive and concave.

Proof. The properties (a) - (d) follow immediately from the corresponding properties for the family of c.n.d.fs.

To see (e), we use criterion (7). Set $\psi(\xi):=\tau_{c} f\left(|\xi|^{2}\right)$. Obviously, $\psi$ is real valued and $\psi(0) \geqslant 0$. Now fix vectors $\xi_{1}, \ldots, \xi_{N} \in \mathbb{R}^{n}$, numbers $\lambda_{1}, \ldots, \lambda_{N} \in \mathbb{C}$ such that $\sum_{j} \lambda_{j}=0$ and assume that $\sum_{j, k} f\left(\left|\xi_{j}-\xi_{k}\right|^{2}+c^{2}\right) \lambda_{j} \bar{\lambda}_{k}>0$. We define $M$ vectors $\eta_{\ell} \in \mathbb{R}^{M}$ by $\eta_{\ell}:=\frac{c}{\sqrt{2}} e_{\ell}\left(e_{\ell}\right.$ is the $\ell$ th unit vector in $\left.\mathbb{R}^{M}\right)$. Then $\left|\eta_{\ell}-\eta_{m}\right|^{2}=c^{2}$ if $\ell \neq m$ and $=0$ if $\ell=m$. By our assumption we can choose $M$ so large that for $\mu_{\ell}=\mu_{m}:=\frac{1}{M}, 1 \leqslant \ell, m \leqslant M$, we find

$$
\sum_{j, k=1}^{N} f\left(\left|\xi_{j}-\xi_{k}\right|^{2}+c^{2}\right) \lambda_{j} \bar{\lambda}_{k} \sum_{\ell \neq m} \mu_{\ell} \bar{\mu}_{m}>-\sum_{j, k=1}^{N} f\left(\left|\xi_{j}-\xi_{k}\right|^{2}\right) \lambda_{j} \bar{\lambda}_{k} \sum_{\ell=1}^{M}\left|\mu_{\ell}\right|^{2} .
$$

Thus,

$$
\begin{aligned}
0 & <\sum_{\ell \neq m} \sum_{j, k=1}^{N} f\left(\left|\xi_{j}-\xi_{k}\right|^{2}+c^{2}\right) \lambda_{j} \bar{\lambda}_{k} \mu_{\ell} \bar{\mu}_{m}+\sum_{\ell=1}^{M} \sum_{j, k=1}^{N} f\left(\left|\xi_{j}-\xi_{k}\right|^{2}\right) \lambda_{j} \bar{\lambda}_{k}\left|\mu_{\ell}\right|^{2} \\
& =\sum_{(\ell, j)} \sum_{(m, k)} f\left(\left|\left(\xi_{j}, \eta_{\ell}\right)-\left(\xi_{k}, \eta_{m}\right)\right|^{2}\right)\left(\lambda_{j} \mu_{\ell}\right) \overline{\left(\lambda_{k} \mu_{m}\right)}
\end{aligned}
$$

Since $\mathbb{R}^{N+M} \ni(\xi, \eta) \mapsto f\left(|(\xi, \eta)|^{2}\right)$ is negative definite and since $\sum_{(\ell, j)} \lambda_{j} \mu_{\ell}=0$, this contradicts $(7)$.

For (f) we use again the criterion (7). Choose $\xi_{1}, \ldots, \xi_{N} \in \mathbb{R}^{n}$ and $\lambda_{1}, \ldots$, $\lambda_{N} \in \mathbb{C}$ with $\sum_{j} \lambda_{j}=0$. For $c \in \mathbb{R}$ we introduce the auxiliary function

$$
\phi(c):=\sum_{j, k=1}^{N}\left[f\left(\left|\xi_{j}-\xi_{k}\right|^{2}+c^{2}\right)-f\left(\left|\xi_{j}-\xi_{k}\right|^{2}\right)\right] \lambda_{j} \bar{\lambda}_{k}
$$

and show that $c \mapsto \phi(c)$ is negative definite. Obviously, $\phi$ is real-valued, $\phi(0)=$ 0 and for all $c_{1}, \ldots, c_{M} \in \mathbb{R}$ and $\mu_{1}, \ldots, \mu_{M} \in \mathbb{C}$ with $\sum_{\ell} \mu_{\ell}=0$ we see

$$
\begin{aligned}
\sum_{\ell, m=1}^{M} \phi\left(\left(c_{\ell}-c_{m}\right)^{2}\right) \mu_{\ell} \bar{\mu}_{m} & =\sum_{\ell, m=1}^{M} \sum_{j, k=1}^{N} f\left(\left|\xi_{j}-\xi_{k}\right|^{2}+\left(c_{\ell}-c_{m}\right)^{2}\right) \lambda_{j} \bar{\lambda}_{k} \mu_{\ell} \bar{\mu}_{m} \\
& =\sum_{(j, \ell)} \sum_{(k, m)} f\left(\left|\left(\xi_{j}, c_{\ell}\right)-\left(\xi_{k}, c_{m}\right)\right|^{2}\right)\left(\lambda_{j} \mu_{\ell}\right) \overline{\left(\lambda_{k} \mu_{m}\right)} \leqslant 0 .
\end{aligned}
$$


The latter follows from (7) and the fact that $\mathbb{R}^{n+1} \ni(\xi, c) \mapsto f\left(|(\xi, c)|^{2}\right)$ is negative definite. Thus, $\phi(c) \geqslant \phi(0) \geqslant 0$ and we conclude that

$$
\sum_{j, k=1}^{N}\left(f-\tau_{c} f\right)\left(\left|\xi_{j}-\xi_{k}\right|^{2}\right) \lambda_{j} \bar{\lambda}_{k}=-\phi(c) \leqslant 0
$$

which, in view of $(7)$, means that $f\left(|\xi|^{2}\right)-\tau_{c} f\left(|\xi|^{2}\right)$ is negative definite.

To see (g) we note that because of (e) $\tau_{c} f \in \mathcal{O}$ for all $c \in \mathbb{R}$, thus $f\left(|\xi|^{2}+\right.$ $\left.c^{2}\right) \geqslant f\left(c^{2}\right)$ which proves that $f$ is increasing. By (f), $f-\tau_{c} f \in \mathcal{O}$, hence $0 \leqslant f\left(|\xi|^{2}\right)+f\left(c^{2}\right)-f\left(|\xi|^{2}+c^{2}\right)$, proving subadditivity. Finally using that $\tau_{c}\left(f-\tau_{d} f\right) \in \mathcal{O}$ we get

$$
0 \leqslant \tau_{c}\left(f-\tau_{d} f\right)\left(d^{2}\right)=2 f\left(c^{2}+d^{2}\right)-f\left(c^{2}+2 d^{2}\right)-f\left(c^{2}\right)
$$

which can be rearranged to give $\frac{1}{2}\left(f\left(c^{2}+2 d^{2}\right)+f\left(c^{2}\right)\right) \leqslant f\left(c^{2}+d^{2}\right)$. Since $f$ is continuous, this mid-point property implies concavity.

We are now ready for the proof of Theorem 2.2.

Proof of Theorem 2.2. The above Lemma shows that $\mathcal{O}$ is a closed convex cone contained in the space $\mathbb{L}=L^{1}\left((0, \infty), e^{-r} d r\right)$. The set

$$
\mathcal{B}=\left\{f \in \mathcal{O}: \int_{0}^{\infty} f(r) e^{-r} d r=1\right\} \subset \mathbb{L}
$$

is a convex base of $\mathcal{O}$. For $f \in \mathcal{B}$ we have

$$
f(r) e^{-r}=f(r) \int_{r}^{\infty} e^{-s} d s \leqslant \int_{r}^{\infty} f(s) e^{-s} d s \leqslant 1 .
$$

Let us show that $\mathcal{B}$ is a relative compact subset of $\mathbb{L}$. We check the conditions of Kolmogorov's compactness criterion for $L^{p}$. First,

$$
\sup _{f \in \mathcal{B}}\|f\|_{\mathbb{L}}=1
$$

Moreover, for all $x, y \geqslant 0$ contained in a bounded set we have

$$
\begin{aligned}
\| f(\bullet+x) & -f(\bullet+y) \|_{\mathbb{L}}=\int_{0}^{\infty}|f(r+x)-f(r+y)| e^{-r} d r \\
& =e^{x \wedge y} \int_{x \wedge y}^{\infty}|f(r+x \vee y-x \wedge y)-f(r)| e^{-r} d r \\
& \leqslant e^{x \wedge y} \int_{0}^{\infty}|f(r+|x-y|)-f(r)| e^{-r} d r \\
& =e^{x \wedge y}\left(e^{|x-y|} \int_{|x-y|}^{\infty} f(r) e^{-r} d r-1\right) \\
& \leqslant e^{x \wedge y}\left(e^{|x-y|}-1\right) \stackrel{|x-y| \rightarrow 0}{\longrightarrow} 0
\end{aligned}
$$


and for all $R>1$ we have by the concavity of $f$ and (25)

$$
\left\|f 1_{(R, \infty)}\right\|_{\mathbb{L}}=\int_{R}^{\infty} f(r) e^{-r} d r \leqslant f(1) \int_{R}^{\infty} r e^{-r} d r=e^{1-R}(R+1) .
$$

Note that (26) and (28) ensure the uniform integrability of the set $\mathcal{B}$, thus $\mathbb{L}_{\text {- }}$ and pointwise convergence coincide on $\mathcal{B}$. This shows that $\mathcal{B}$ is closed, hence compact.

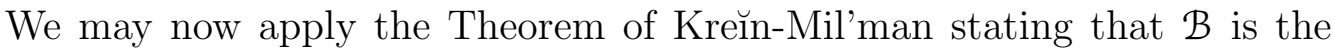
closed convex hull of its extremal points. Literally as in Harzallah [8] we find that the extremals of $\mathcal{B}$ are $\left\{1, x, \frac{1+\gamma}{\gamma}\left(1-e^{-\gamma x}\right), 0<\gamma<\infty\right\}$; hence, every $f \in \mathcal{O}$ is of the form

$$
f(x)=c\left(\alpha+\beta x+\int_{0+}^{\infty}\left(1-e^{-\gamma x}\right) \frac{1+\gamma}{\gamma} \mu(d \gamma)\right)
$$

for some $c, \alpha, \beta \geqslant 0$, and a measure $\mu$ such that $\alpha+\beta+\mu((0, \infty)) \leqslant 1$.

\section{References}

[1] Alzer, H. and C. Berg: Some classes of completely monotonic functions. Ann. Acad. Sci. Fenn. 27 (2002), $445-460$.

[2] Andrews, G. E., Askey, R. and R. Roy: Special Functions. Encycl. Math. Appl. Vol. 71. Cambridge: Cambridge University Press 1999.

[3] Berg, C.: Integral representation of some functions related to the Gamma function. Mediterranean J. Math., to appear.

[4] Berg, C. and G. Forst: Potential Theory on Locally Compact Abelian Groups. Ergeb. Math. Grenzgeb. Bd. 87. Berlin: Springer-Verlag 1975.

[5] Berg, C. and H. L. Pedersen: A completely monotone function related to the Gamma function. J. Comp. Appl. Math. 133 (2001), 219 - 230.

[6] Beurling, A. and J. Deny: Dirichlet Spaces. Proc. Natl. Acad. Sci. U.S.A. 45 (1959), $208-215$.

[7] Farkas, W. and H.-G. Leopold: Characterisations of function spaces of generalised smoothness. Ann. Mat. Pura Appl., to appear.

[8] Harzallah, Kh.: Fonctions opérant sur les fonctions définies-négatives. Ann. Inst. Fourier 17 (1967)(1), 443 - 468.

[9] Hörmander, L.: Linear Partial Differential Operators. Grundlehren Math. Wiss. Bd. 116. Berlin: Springer-Verlag 1963.

[10] Hörmander, L.: The Analysis of Linear Partial Differential Operators II. Grundlehren Math. Wiss. Bd. 257. Berlin: Springer-Verlag 1983.

[11] Ismail, M. E. H.: Bessel functions and the infinite divisibility of the Student t-distribution. Ann. Probab. 5 (1977), 582 - 585. 
[12] Ismail, M. E. H., Integral representations an complete monotonicity of various quotients of Bessel functions. Can. J. Math. 29 (1977), 1198 - 1207.

[13] Ismail, M. E. H. and C. Ping May: Special functions, infinite divisibility and transcendental equations. Math. Proc. Camb. Phil. Soc. 85 (1979), 453 - 464.

[14] Ismail, M. E. H.: Complete monotonicity of modified Bessel functions. Proc. Am. Math. Soc. 108 (1990), 353 - 361.

[15] Ismail, M. E. H. and K. S. Miller: An infinitely divisible distribution involving modified Bessel functions. Proc. Am. Math. Soc. 85 (1982), 233 - 238.

[16] Jacob, N.: Pseudo Differential Operators and Markov Processes. Vol. 1: Fourier Analysis and Semigroups. London: Imperial College Press 2001.

[17] Kahane, J.-P.: Sur les fonctions de type positif et de type négatif. In: Seminar on Harmonic Analysis 1978-79. Publ. Math. Orsay 79.7, Univ. Paris XI, Orsay 1979 , pp. $21-37$.

[18] Masja (Maz'ya), V. G. and J. Nagel: Über äquivalente Normierung der anisotropen Funktionalräume $H^{\mu}\left(\mathbb{R}^{n}\right)$. Beitr. Anal. 12 (1978), 7 - 17.

[19] Nagel, J.: Äquivalente Normierungen in Funktionalräumen (in Russian). Vestn. L.G.U. 7 (1974), $41-47$.

[20] Nagel, J.: Verallgemeinerte Slobodezki-Räume auf der Halbachse. Beitr. Anal. 15 (1981), $61-70$.

[21] Schilling, R. L.: Zum Pfadverhalten von Markovschen Prozessen, die mit LévyProzessen vergleichbar sind. Dissertation, Universität Erlangen 1994.

[22] Schilling, R. L.: Subordination in the sense of Bochner and a related functional calculus. J. Aust. Math. Soc. Ser. A 64 (1998), 368 - 396.

[23] Schoenberg, I. J.: Metric spaces and completely monotone functions. Ann. Math. 39 (1938), $811-842$.

[24] Song, R.: Private Communication, September 2004.

[25] Triebel, H.: The Structure of Functions. Monogr. Math. Vol. 97. Basel: Birkhäuser Verlag 2001.

Received 04.05.2004 